\title{
Apoyo social, estrés y autoestima en mujeres de familias monoparentales y biparentales
}

\author{
Social support, stress and self-esteem in women from single-parent \\ and bi-parent families
}

\author{
René Landero Hernández ${ }^{1}$ \\ Mónica T. González Ramírez² \\ Universidad Autónoma de Nuevo León, México \\ (Rec: Diciembre 2010 - Acep: Abril 2011)
}

\begin{abstract}
Resumen
El objetivo del presente estudio es describir las características de las mujeres por tipo de familia y comparar los puntajes de apoyo social, estrés y autoestima en mujeres de familias biparentales versus mujeres de familias monoparentales. La muestra fue por conveniencia. Fueron entrevistados en sus casas un total de 210 mujeres con hijos, 105 de familias biparentales y 105 de familias monoparentales, las cuales tienen en promedio 39.9 años de edad y 2.1 hijos. Los resultados principales utilizando la U de Mann-Whitney para evaluar las diferencias entre familias, indican que los puntajes de las mujeres de familias monoparentales, en apoyo social y autoestima son menores que los de las mujeres de familias biparentales, y mayores en estrés.
\end{abstract}

Palabras clave: mujeres, familias monoparentales, familias biparentales, apoyo social, estrés, autoestima.

\begin{abstract}
The objectives of the current study were to describe the characteristics of women from single-parent families and bi-parent families; and to compare their scores of social support, stress and self-esteem. We worked with a nonprobabilistic sample of 210 mothers, 105 from bi-parent families and 105 from single-parent families. Participants were interviewed in their houses. The mean age was 39.9 years old, and the children average was 2.1. Main results using Mann-Whitney U to compare differences between types of families showed that single woman score lower on scales of social support and self-esteem, and higher on scale of stress than women from bi-parent families.

Key words: women, single-parent families, bi-parent families, social support, stress, self-esteem.
\end{abstract}

Correspondencia a: René Landero Hernández. Dirección postal: Mutualismo 110, Col. Mitras Centro, 64460 Monterrey, N. L., México. Tel: (81) 8333-8233, fax: (81) 8333-8222.E-mail: rlandero1_mx@yahoo.com.mx.

2 Mónica T. González Ramírez. Dirección postal: Mutualismo 110, Col. Mitras Centro, 64460 Monterrey, N. L., México. Tel: (81) 8333-8233, fax: (81) 8333-8222. E-mail: monygzz77@yahoo.com. 


\section{Introducción}

Uno de los cambios más significativas en las últimas décadas en la composición de los hogares, es el incremento de las familias encabezadas por mujeres al pasar de poco menos de uno de cada ocho en 1976 (13.5\%), a más de uno de cada cinco hogares $(20.6 \%)$ en 2000 . Así, en la actualidad el número de unidades domésticas encabezadas por mujeres es de 4.6 millones, cuando en 1990 ascendía a 2.8 millones (CONAPO, 2002). En la mayoría de los hogares encabezados por mujeres, ellas son viudas, divorciadas o separadas. Esta condición de la mujer de vivir sin su cónyuge, deriva en una familia monoparental.

Las familias monoparentales son muy diversas entre sí, tanto por su origen, edad, género, recursos de partida o circunstancias vitales. Con respecto a su origen, una familia monoparental puede derivarse de tres situaciones tradicionales: la maternidad biológica o adoptiva en solitario (madre soltera), la muerte de uno de los cónyuges (viudez), o bien a partir de la separación (de facto), o el divorcio (de jure). Estas circunstancias configuran distintas trayectorias familiares, que propician una desigualdad social y psicológica (Landero, 2001, 2005; Rodríguez, 1997).

De acuerdo a los datos del INEGI (2005) para el año 2000, las personas separadas y divorciadas se han incrementado en las últimas décadas, en el periodo $1990-2000$ pasaron de un 3.8\% a un 7\%. De acuerdo con la información de la Encuesta Nacional de Ocupación y Empleo 2008 (INEGI, 2009), en el país la población de 12 años o más ascendía a 81.6 millones: 38.6 millones de hombres y 43 millones de mujeres. De las personas de esta edad, 37 de cada 100 son solteras; 54 viven en pareja -casadas o en unión libre- y 9 están separadas, divorciadas o viudas.

Tanto hombres como mujeres enfrentan dificultades después de la disolución o ruptura del vínculo conyugal (ya sea por separación, divorcio o viudez). En el caso de las mujeres, estas dificultades se extienden a lo emocional, lo psicológico y lo económico (Valdés, 2004; Zúñiga \& Ribeiro, 2005). Estos problemas durante la ruptura conyugal y después de ella, son factores generadores de estrés, depresión y de otros problemas de salud, que afectan a su condición social y calidad de vida (Landero, 2002; Landero, Estrada \& González, 2009). Además, en la ruptura conyugal también se pierde el apoyo social de la pareja. Durante y después de la ruptura conyugal, esta afecta en forma diferente, tanto al nivel de estrés y autoestima de hombres y mujeres, así como el apoyo provisto por la pareja, amigos y las familias de los cónyuges.

El apoyo social es particularmente importante en el estudio de padres divorciados, porque este puede cambiar la red social, reduciendo su densidad y duración de los lazos sociales (Sprecher et al., 2006, en DeGarmo, Patras \& Eap, 2008).

Por otra parte, se ha demostrado que la ruptura conyugal o del matrimonio, es uno de los eventos más estresantes (Bloom, Asher y White, 1978; Verbrugge, 1979); lo que genera un aumento en los niveles de estrés y es frecuente que este continúe durante algún tiempo después del divorcio (Hetherington, Cox \& Cox, 1979; Boot \& Amato, 1991); también, el divorcio es asociado con altas tasas de desordenes físicos y mentales (Bloom, Asher \& White, 1978). La disolución marital incrementa el riesgo de problemas de salud físicos y mentales (Prigerson, Maciejewski \& Rosenheck, 1999). Sin embargo, algunos estudios han reportado que las mujeres experimentan mayormente la ansiedad con la separación y reciben menor apoyo instrumental y emocional (Deater-Deckard, Scarr, McCartney \& Eisenberg, 1994; Wille, 1998). En el caso del apoyo social, este se relaciona en forma negativa con el estrés (Landero \& González, 2004). Además, el apoyo (percibido) provisto por el cónyuge, se relaciona positivamente con la calidad de la relación marital y negativamente con sintomatología depresiva y con el estrés percibido (Dehle, Larsen \& Landers, 2001).

Los estudios también han arrojado indicios en torno a que las personas con pareja estable, amigos y familia que les proporcionen recursos materiales y psicológicos, tienen mejor salud que aquellos con un contacto social pobre o en crisis (Cohen $\&$ Ashby, 1985). Es decir, una adecuada relación de pareja constituye un importante soporte social en la etapa del ciclo vital (Perales, Sogi \& Morales, 2003). Como lo señalan otros autores, el matrimonio se asocia a una mejor salud (Verbrugge, 1979) y a menos depresión (Ross \& Mirowsky, 1989), o como lo reconocen Waldron, Hughes y Brooks (1996), el matrimonio juega un papel de protección en la salud de las mujeres, principalmente porque este ofrece apoyo social y recursos financieros. Otros estudios observaron que por su condición de "madres solas", estas tienen desventajas económicas y de salud (Burstrom, Diderichsen, Shouls \& Whitehead, 1999). Es así que su estatus de salud es más pobre comparadas con las madres viviendo con su cónyuge (Benzeval, 1998; Landero, Estrada \& González, 2009). 
De acuerdo a los resultados encontrados por Cairney, Boyle, Offord y Racine (2003), las madres casadas comparadas con las madres solas (sin cónyuge), estas últimas reportan mayores niveles de estrés, mayor número de adversidades en la niñez, y también, menores niveles de apoyo social percibido, involucramiento social y frecuencia de contactos con amigos y familia. Varias investigaciones han subrayado la trascendencia de dimensiones funcionales como el apoyo emocional y la disponibilidad de vínculos sociales de confianza, con quienes poder compartir sentimientos y experiencias vitales (Hernández \& Gilbert, 1987; Vega, Kolody, Valle \& Weir, 1991).

El apoyo social es un constructo multidimensional que con frecuencia se define según los propósitos de cada estudio. Este puede referirse al número o densidad de la red social de apoyo, a la calidad de ésta, a la percepción subjetiva respecto de que ciertas conductas brindan apoyo y al grado de intimidad con la pareja, y puede aludir a aspectos emocionales o a instrumentales (Landero \& González, 2006). Frente a estas definiciones, se remarcan los aspectos funcionales del apoyo social, centrándose en este caso en el tipo de recursos aportados por los lazos sociales y las funciones que cumplen, destacando diversas dimensiones o aspectos del mismo, como el apoyo emocional, de estima y material (Barrón, 1990a, en Barrón, 1996).

Analizando los diversos estudios, lo que parece claro es que los efectos directos aparecen en investigaciones que usan medidas estructurales del apoyo social, cuando lo que se evalúa es integración en una red social, pertenencia a grupos, estado civil, etc. En cambio, los efectos protectores se evidencian cuando se conceptualiza el apoyo social según sus dimensiones funcionales, es decir, cuando se evalúa la disponibilidad de recursos que ayudan a los individuos a responder a los eventos vitales estresantes (Martín, Chacón \& Martínez, 1988).

Es por lo anterior que existen diferentes medidas para evaluarlo, de acuerdo a su conceptualización y/o perspectiva. Cohen y Syme, 1985; House y Kan, 1985 (en Sherbourne \& Stewart, 1991), señalan que el más esencial aspecto del apoyo social es la disponibilidad percibida de apoyo funcional, que se refiere al grado en el cual las relaciones interpersonales sirven a una particular función. Las funciones más frecuentemente citadas son: 1) apoyo emocional, el cual refiere a cuidados, amor y empatía; 2) apoyo instrumental, referido también como tangible; 3) apoyo informativo, orientación o retroalimentación que puede proveer una solución a un problema; 4) valorativo, el cual involucra información relevante para una auto evaluación; y 5) interacción social, el cual implica tiempo utilizado con otros en actividades de tiempo libre y recreativas (House, 1981; Wills, 1985, en Sherbourne \& Stewart, 1991). En el presente estudio se utiliza la medida de apoyo social percibido.

Hablando del estrés, existen estudios que ponen de manifiesto que esta variable tiene un impacto negativo sobre la salud, ya que disminuye la competencia inmunológica del individuo sometido a situaciones estresantes o estresores (Landero \& González, 2002). Además, el estrés parece jugar un papel importante en la explicación de las diferencias en el bienestar psicológico entre madres solas y casadas (Cairney et al., 2003).

Musitu y Cava (2001) distinguen dos tipos de estresores: los normativos y los no normativos. Los estresores de tipo normativo son aquellas situaciones que ocurren a la mayor parte de las familias en un momento concreto de su ciclo de vida familiar. Por ejemplo, las familias con hijos pequeños tienen estresores relacionados con el mayor número de tareas domésticas que atender, y las familias con hijos adolescentes suelen tener numerosos conflictos con sus hijos como consecuencia de sus deseos de autonomía e independencia. Se trata, por tanto, de estresores que están tipificados, y, en consecuencia, son parte de la cultura. Son estresores esperados, porque aunque se trata de situaciones nuevas, suelen ser "advertidas" previamente por familiares o amigos, o, simplemente, las hemos observado en otras familias de nuestro entorno.

Por el contrario, las situaciones estresantes no normativas son aquellas que no tienen por qué ocurrir a todas las familias, y tampoco a una determinada edad. Así, por ejemplo, la jubilación es un suceso normativo si se produce entre los 60 y los 70 años, pero una jubilación anticipada a los 40 años sería un suceso no normativo. Los eventos no normativos son, por tanto, situaciones inesperadas, de aparición generalmente abrupta y con la suficiente importancia como para provocar cambios en el funcionamiento familiar. Algunos otros ejemplos de estresores no normativos son la presencia en la familia de un miembro con problemas de drogodependencia, el abandono de la familia de alguno de los esposos o la muerte prematura e inesperada de algún miembro de la familia (Musitu \& Cava, 2001).

Finalmente, Lila, Buelga y Musitu (2006) indican que la autoestima, además de ser esencial para que el individuo reconozca y afronte los estímulos estresantes, es un recurso amortiguador del estrés. 
En estudios donde se evaluaron exclusivamente mujeres, se ha identificado una relación negativa entre autoestima y estrés (Landero \& González, 2002; Landero \& González, 2004).

A pesar de los datos mencionados y de que el estudio del estrés, la autoestima y el apoyo social no es nuevo, las características de las familias monoparentales, la variedad de características de dichas familias (viudas, divorciadas, madres solteras), los cambios generacionales, entre otras variables, hacen que las investigaciones enfocadas en esta subpoblación sea vigente. Profundizar en las condiciones particulares de dichas familias y compararlas con otros tipos de familias permitirá contribuir no solo a la descripción de la subpoblación, sino también plantear las bases para el trabajo específico con estas familias. Es por lo anterior que los objetivos del presente estudio son describir las características de las mujeres por tipo de familia y comparar los puntajes de apoyo social, estrés y autoestima en mujeres de familias biparentales versus mujeres de familias monoparentales.

\section{Método}

Para el cumplimiento de los objetivos planteados se trabajó con un diseño transversal. Las características de la población e instrumento se presentan en este apartado. La muestra fue por conveniencia de 210 mujeres con hijos, 105 mujeres de familias biparentales y 105 mujeres de familias monoparentales, las cuales se entrevistaron en sus casas.

\section{Instrumentos de evaluación}

El cuestionario MOS de Apoyo Social (Sherbourne \& Stewart, 1991), consta de 20 ítems con 5 opciones de respuesta (de 1 a 5 ). La consistencia interna medida por los autores con el alfa de Cronbach es mayor de .91 en la puntuación total. La fiabilidad test-retest es de .78 (Sherbourne \& Stewart, 1991). En otro estudio, el coeficiente alfa de Cronbach para las dimensiones fue de .77 a .89 , y en la escala global de 93 (Landero \& González, 2006). El rango de la puntuación total de los 19 ítems es de 19-95; a mayor puntuación en el cuestionario, mayor apoyo percibido. Los 19 ítems constituyen 4 dimensiones del apoyo social funcional: apoyo emocional/informacional (ítems 3, 4, 8, 9, 13, 16, 17 y 19), apoyo instrumental (ítems 2, 5, 12 y 15), interacción social positiva (ítems $7,11,14$ y 18) y apoyo afectivo (ítems 6, 10 y 20).
La Escala de Estrés Percibido (Perceived Stress Scale, PSS) fue elaborada por Cohen, Kamarak y Mermelstein (1983) para evaluar el grado en que las situaciones de la vida son valoradas como estresantes, obteniendo un alfa de Cronbach de .75. En este estudio se utilizará la adaptación cultural realizada en México por González y Landero (2007), la cual cuenta con 14 ítems y una consistencia interna $(\alpha=.83)$ en la muestra de México, misma que se utilizó para confirmar la estructura factorial de la escala original, utilizando un análisis factorial confirmatorio. La puntuación va de nunca -0- a muy a menudo -4-, invirtiéndose la puntuación en los ítems negativos. La mayor puntuación corresponde a mayor estrés percibido.

La escala de autoestima de Rosenberg (1989) evalúa el nivel de satisfacción con uno mismo. Esta escala está conformada por 10 ítems, cinco de ellos enunciados en forma positiva y cinco en forma negativa. La puntuación total oscila entre 10 y 40; a mayor puntuación, mayor es la autoestima. En la presente investigación se utilizó la versión española de Martín-Albo, Núñez, Navarro y Grijalvo (2007), quienes obtuvieron en la primera y segunda aplicación un coeficiente alfa de .85 y .88 , respectivamente. El valor de la correlación test-retest fue de .84 .

En cuanto al resto de las variables, la condición laboral se midió con la pregunta ¿Tiene actualmente un trabajo remunerado?, con respuesta de sí o no; la edad se midió con el número de años cumplidos, la escolaridad con el número de años terminados de estudio, el número de hijos se refiere al número de hijos viviendo en el hogar y el tamaño del hogar es el número de personas que residen en la vivienda.

\section{Resultados}

Como primer paso se valoró la consistencia interna de las escalas utilizadas, a través del alfa de Cronbach, encontrando niveles aceptables en todas ellas. Los resultados de este análisis se presentan en la tabla 1. El coeficiente obtenido de Cronbach para las dimensiones de apoyo social va de .77 a .88 , y el obtenido en la escala global fue .95. En la escala de autoestima se obtuvo un coeficiente de .79 y en la de estrés .82 .

Se procedió a evaluar la normalidad de las variables psicosociales con la prueba de KolmogorovSmirnov, los resultados indican que las variables no se comportaban con normalidad $(\mathrm{p}<.05)$, además de los gráficos y diagramas descriptivos; por lo que se decidió usar la prueba estadística $U$ de 
Tabla 1. Consistencia interna de las escalas y dimensiones.

\begin{tabular}{ll}
\hline Escalas & $\alpha$ \\
\hline Escala de Apoyo Social & .95 \\
Dimensión: Apoyo Emocional & .93 \\
Dimensión: Apoyo Instrumental & .77 \\
Dimensión: Interacción social positiva & .88 \\
Dimensión: Apoyo Afectivo & .87 \\
Autoestima & .79 \\
Estrés & .82 \\
\hline
\end{tabular}

Tabla 2. Descripción de las variables demográficas de la muestra por tipo de familia $(\mathrm{n}=210)$.

\begin{tabular}{lllll}
\hline Variable & Familia & Media & D.T. & U de Mann-Whitney \\
\hline \multirow{2}{*}{ Edad } & Biparental & 39.49 & 8.87 & \multirow{2}{*}{$\mathrm{Z}=-.516, \mathrm{p}=.606$} \\
& Monoparental & 40.37 & 12.63 & \\
\hline \multirow{2}{*}{ Escolaridad } & Biparental & 11.81 & 3.29 & \multirow{2}{*}{$\mathrm{Z}=-.572, \mathrm{p}=.567$} \\
& Monoparental & 11.53 & 3.48 & \\
\hline \multirow{2}{*}{ Número de hijos } & Biparental & 2.34 & .87 & \multirow{2}{*}{$\mathrm{Z}=-3.981, \mathrm{p}=.001$} \\
& Monoparental & 1.88 & .94 & \\
\hline \multirow{2}{*}{ Tamaño del hogar } & Biparental & 4.47 & .97 & \multirow{2}{*}{$\mathrm{Z}=-6.196, \mathrm{p}=.001$} \\
& Monoparental & 3.47 & 1.35 & \\
\hline
\end{tabular}

Mann-Whitney para evaluar las diferencias entre familias monoparentales y biparentales.

Los datos observados en la tabla 2, indican que las mujeres de familias monoparentales tienen en promedio alrededor de 1 año más de edad que las mujeres de familias biparentales, y un nivel de escolaridad similar. En cambio, en el tamaño de la familia (número de personas que viven en la casa) y número de hijos, es menor el promedio en las monoparentales. De acuerdo a los datos del INEGI (2001), para el 2000, el tamaño de las familias monoparentales era de 3.2 miembros, mientras que el de las biparentales de 4.1, los cuales son semejantes a los aquí presentados, y son significativamente diferentes entre ambos tipos de familia; no así, la edad y escolaridad.

En la siguiente tabla 3 se presenta la información de la condición de trabajo de las mujeres de la muestra por tipo de familia. El $76.2 \%$ de las mujeres de familias monoparentales trabajan (trabajo remunerado), en cambio, el $55.2 \%$ de las mujeres de familias biparentales trabaja fuera del hogar. De acuerdo a la Encuesta Nacional de Empleo (INEGISTPS, 2001), en el 2000 el porcentaje de mujeres casadas y en unión libre que trabajaban fue de 46.5; y la población divorciada tenía una alta participación laboral (73.5\%) (INEGI, 2003).

Como se puede observar en la tabla 4, las medias de las variables psicosociales analizadas, en el caso de las mujeres de familias monoparentales son menores en apoyo social y autoestima, y mayores en estrés. Además, hay diferencia significativa entre los tipos de familia de acuerdo a los resultados de la U de Mann-Whitney.

Tabla 3. Descripción del trabajo remunerado por tipo de familia $(\mathrm{n}=210)$.

\begin{tabular}{cccc}
\hline \multirow{2}{*}{ Tipo de Familia } & \multicolumn{2}{c}{ Trabajo Remunerado } & \multirow{2}{*}{ Total } \\
\cline { 2 - 3 } & Sí & No & \\
\hline Biparental & $47(44.8 \%)$ & $58(55.2 \%)$ & 105 \\
Monoparental & $80(76.2 \%)$ & $25(23.8 \%)$ & 105 \\
\hline
\end{tabular}


Tabla 4. Descripción de las variables psicosociales por tipo de familia $(\mathrm{n}=210)$.

\begin{tabular}{ccccc}
\hline Variable & Familia & Media & D.T. & U de Mann-Whitney \\
\hline \multirow{2}{*}{ Apoyo Social } & Biparental & 77.09 & 14.26 & \multirow{2}{*}{$\mathrm{Z}=-3.063, \mathrm{p}=.002$} \\
& Monoparental & 40.37 & 15.79 & \multirow{2}{*}{$\mathrm{Z}=-3.131, \mathrm{p}=.002$} \\
\hline \multirow{2}{*}{ Estrés } & Biparental & 20.50 & 6.62 & \\
& Monoparental & 23.50 & 6.75 & $\mathrm{Z}=-2.145, \mathrm{p}=.032$ \\
\hline \multirow{2}{*}{ Autoestima } & Biparental & 32.72 & 4.09 & \\
& Monoparental & 31.41 & 4.22 &
\end{tabular}

\section{Discusión y conclusiones}

Los resultados generales obtenidos de acuerdo al tipo de familia, indican diferencias significativas en el tamaño del hogar y número de hijos, teniendo las familias monoparentales el menor número, lo cual coincide con la información de las estadísticas nacionales (INEGI, 2001) y de otros estudios (McLanahan, Wedemeyer \& Adelberg, 1981; Anson, 1988; Landero, 2001, 2005; Landero \& González, 2006). En edad y escolaridad no se encontraron diferencias entre los tipos de familia, lo cual es congruente con otros estudios (Verbrugge, 1979; INEGI, 2001; McLanahan, Wedemeyer \& Adelberg, 1981; Landero \& González, 2006).

En cuanto a la actividad económica, las mujeres de familias monoparentales tienen una participación mayor (76\%), esta es similar a lo encontrado en estudios previos (INEGI, 2003; Landero, 2002, 2005; Landero \& González, 2006; Zúñiga \& Ribeiro, 2005). Las personas que viven una ruptura conyugal (divorciadas o separadas), por su particular curso de vida, tienen responsabilidades económicas que solventar, como su sostenimiento personal y el de su descendencia, sobre todo si no reciben dinero de su ex cónyuge (pensión). El nivel de vida de las personas involucradas disminuye con frecuencia después de la ruptura conyugal y en el caso de las mujeres, generalmente sufren una pérdida financiera más importante que la de sus ex cónyuges, es decir, se empobrecen (Brown \& Moran, 1997; Holden \& Smock, 1991). Por este motivo, la mayoría de ellas tiene la necesidad de desarrollar algún tipo de actividad económica. En esta misma línea, McLanahan (1983) encontró que la separación y el divorcio son estresores, así como la reducción del ingreso y la reubicación del lugar donde vivir.

Asimismo, los resultados en las variables psicosociales anteriormente mencionados, indican menor apoyo social y autoestima, y un mayor estrés en las mujeres de familias monoparentales con respecto a las mujeres de familias biparentales. Esto también coincide con los resultados de otros estudios (Booth \& Amato, 1991; Landero \& González, 2006; Zúñiga \& Ribeiro, 2005).

En lo referente a la diferencia en los puntajes de apoyo social por tipo de familia, esta se debe principalmente a las dimensiones de vínculos sociales (relaciones personales, apoyo social y actividad sexual, y afectivo). Como se mencionó previamente, las relaciones sociales son uno de los aspectos que más fácilmente se deteriora después de la disolución o ruptura del vínculo conyugal. Algunas de las implicaciones sociales del divorcio (y la separación) para con los hijos y la esposa de acuerdo a Lamb (1977), son aparte de la pérdida del ingreso del cónyuge, la pérdida de apoyo emocional y el aislamiento. Los padres "solos" tienden a estar más aislados que los padres casados, reciben menos apoyo emocional y son menos estables sus redes sociales (McLanahan, Wedemeyer \& Adelberg, 1981; Weinraub \& Wolf, 1983). Asimismo, uno de los efectos indirectos de la ausencia del padre en el incremento de los estresores emocionales y financieros (Wallerstein \& Kelly, 1975).

De tal suerte, frente a los problemas resentidos después de la ruptura y frente a las condiciones en las que viven ciertas personas - en particular las madres que viven solas - ; y aunque esta soledad suele ser temporal, es una etapa de aislamiento producto de la separación o ruptura, en la cual los lazos afectivos y las relaciones significativas se rompen o entran en conflicto, por lo que es necesario buscar más vínculos que puedan aportar y dar apoyo, para superar la crisis y adaptarse a las nuevas circunstancias, sin embargo, una cosa es estar solo y otra sentirse solo. La soledad física es la falta de compañía, es estar solo. Pero la persona que está sola, no necesariamente tiene por qué sentirse sola; es decir, la presencia de otra persona anula el estado de soledad, pero no quiere decir que no tenga un sentimiento de soledad. La buena percepción del apoyo social ayuda a que la persona no se sienta sola; debe haber compañerismo y buena correspondencia entre las personas; esto es, 
apoyo recíproco, pero si no existe esta reciprocidad el sentimiento de soledad se mantiene (Gracia, Herrero \& Musitu, 1995).

Russel y Cutrona (1991) reportan que un déficit en la cantidad -y calidad- de apoyo social se asocia a un incremento en los síntomas depresivos y que las personas que tienen más relaciones interpersonales son menos vulnerables a los efectos del estrés o a las situaciones críticas que van enfrentando en la vida. En el caso de las mujeres, el hecho de estar casadas era un factor protector para el desarrollo de la depresión (Zung, Broadhead \& Roth, 1993).

Es importante continuar en el análisis de estas y otras variables como las relaciones sociales, el apoyo social, y su efecto en la salud y calidad de vida de las mujeres que encabezan familias, sobre todo, de los diferentes tipos de familias monoparentales (simples o de un núcleo, extensas y complejas) y de acuerdo a su estado civil (separadas, divorciadas, viudas y madres solteras). Asimismo, diferenciar el posible efecto del "padre ausente" en las familias biparentales, ya que, aunque existe la pareja, puede haber poca o nula participación en las actividades de la familia, conflictos o problemas de comunicación.

\section{Referencias}

Anson, O. (1988). Living arrangements and women's health. Social Science \& Medicine, 26 (2), 201-208.

Barrón, A. (1996). Apoyo social. Aspectos teóricos y aplicaciones. España: Siglo XXI.

Benzeval, M. (1998). The self-reported health status of lone parents. Social Science \& Medicine, 46(10), 1337-1353.

Bloom, B. L., Asher, S. J. \& White, S. W. (1978). Marital disruption as a stressor: A review and analysis. Psychological Bulletin, 85(4), 867-894.

Booth, A. \& Amato, P. (1991). Divorce and Psychological Stress. Journal of Health and Social Behavior, 32(4), 396-407.

Brown, G. W. \& Moran, P. (1997). Single mothers, poverty and depression. Psychological Medicine, 27, 21-33.

Burstrom B., Diderichsen F., Shouls, S., \& Whitehead M. (1999). Lone mothers in Sweden: trends in health and socioeconomic circumstances, 1979-1995. Journal of Epidemiology Соттиnity Health, 53(12), 750-756.

Cairney, J., Boyle, M., Offord, D. R. \& Racine, Y. (2003). Stress, social support and depression in single and married mothers, Social Psychiatry \& Psychiatric Epidemiology, 38(8), 442-449.

Cohen, S. Kamarak, T. \& Mermelstein, R. (1983). A global measure of perceived stress. Journal of Health and Social Behaviour, 24, 385-396.

Cohen, S. y Ashby, T. (1985). Stress, Social Support and the Buffering Hypothesis. Psychological Bulletin, 98(2), 310- 357.

CONAPO (2002). Comunicación social, 2 de marzo de 2002. Consejo Nacional de Población. Revisado el 28 de abril de 2009. Disponible en: http://www.conapo.gob.mx/ prensa/2002/2002mzo02.htm

Deater-Deckard, K., Scarr, S., McCartney, K., \& Eisenberg, M. (1994). Paternal separation anxiety: Relationships with pa- renting stress, child-rearing attitudes, and maternal anxieties. Psychological Science, 5(6), 341-346.

DeGarmo, D., Patras, J., \& Eap, S. (2008). Social Support for Divorced Fathers' Parenting: Testing a stress buffering model. Family Relations, 57(1), 35-48.

Dehle, C., Larsen, D. \& Landers, J. E. (2001). Social Support in Marriage. The American Journal of Family Therapy, 29, 307-324.

González, M. \& Landero, R. (2007). Factor Structure of the Perceived Stress Scale (PSS) in a Sample from Mexico. The Spanish Journal of Psychology, 10 (1), 199-206.

Gracia, E., Herrero, J. \& Musitu, G. (1995). El apoyo social. Barcelona: PPU.

Hernández, E. \& Gilbert, L. A. (1987). Social support networks for parenting and psychological well-being among dual-earner Mexican-American families. Journal of Community Psycho$\log y, 15(2), 176-185$.

Holden, K., \& Smock, P. (1991). The Economic Costs of Marital Dissolution: Why Do women Bear a Disproportionate Cost?, Annual Review Sociology, 17, 15-78.

INEGI-STPS (2001). Encuesta Nacional de Empleo 2000. Aguascalientes. Instituto Nacional de Estadística, Geografía e Informática/Secretaría del Trabajo y Previsión Social.

INEGI (2001). Indicadores sociodemográficos de México (19302000). Aguascalientes. Instituto Nacional de Estadística, Geografía e Informática.

INEGI (2003). Mujeres y Hombres en México 2003 ( $7^{\mathrm{a}}$ edición). Información digital. Aguascalientes. Instituto Nacional de Estadística, Geografía e Informática/Instituto Nacional de las Mujeres.

INEGI (2005). Estadísticas de Matrimonios y Divorcios 2003. Cuaderno núm. 10. Aguascalientes. Instituto Nacional de Estadística, Geografía e informática.

INEGI (2009). Estadísticas a propósito del 14 de febrero, Matrimonios y Divorcios en México. Aguascalientes. Instituto Nacional de Estadística, Geografía e informática. Consultado el 27 de marzo de 2009, disponible en: http://200.23.8.5/ inegi/contenidos/espanol/prensa/contenidos/estadisticas/2009/ matrimonios09.asp?s=inegi\&c $=2680 \&$ ep $=9$

Lamb, M. (1977). Father-infant and mother-infant interaction in the first year of life. Child Development, 48, 167-181.

Landero, R. (2001). Las familias monoparentales: sus características y tipología. Revista de ciencias sociales, 90-91, 9-23.

Landero, R. (2002). Familias y familias monoparentales: su formación, diversidad y condición social. En: Raúl López E. (ed.). La pobreza en Monterrey: los recursos económicos de las unidades domésticas. UANL, 235-289.

Landero, R. (2005). Origen, definición y diversidad de la monoparentalidad. En Landero, R. (Ed.) (2005). Ruptura conyugal y monoparentalidad. Dificultades afrontadas y apoyo social. Facultad de Psicología, UANL, 23-54.

Landero, R. \& González, M. (2002). Determinantes psicosociales del estrés en amas de casa. Psicología y Salud, 12 (2), 279-288.

Landero, R. \& González, M.T. (2004). Variables psicosociales como predictoras de la salud en mujeres. Revista de Psicología Social, 19 (3), 255-264.

Landero, R. \& González, M.T. (2006). Apoyo social en mujeres de familias monoparentales y biparentales. Psicología y Salud, $16(2), 149-157$.

Landero, R., Estrada, B. \& González, M.T. (2009). Depression and Quality of Life for Women in Single-parent and Nuclear Families. The Spanish Journal of Psychology, 12 (1), 171-183.

Lila, M., Buelga, S. \& Musitu, G. (2006). Las relaciones entre padres e hijos en la adolescencia. Madrid: Pirámide.

McLanahan, S., Wedemeyer, N. \& Adelberg, T. (1981). Network Structure, Social Support, and Psychological Well-Being in the 
Single-Parent Family. Journal of Marriage and the Family, 43(3), 601-612.

McLanahan, S. (1983). Family structure and stress: A longitudinal comparison of two- parent and female-headed families. Journal of Marriage and the Family, 45, 347-357.

Martín, A., Chacón, F. y Martínez, M. (1988). Psicología Comunitaria. Barcelona: Textos Visor.

Martín-Albo, J., Núñez, J., Navarro, J. \& Grijalvo, F. (2007). The Rosenberg Self-Esteem Scale: Translation and Validation in University Students. The Spanish Journal of Psychology, 10(2), 458-467.

Musitu, G. \& Cava, M. J. (2001). La familia y la educación. Barcelona: Octaedro.

Perales, A., Sogi, C. \& Morales, R. (2003). Estudio comparativo de salud mental en estudiantes de medicina de dos universidades estatales peruanas, Anales de la Facultad de Medicina, 64(4), 239-246.

Prigerson, H. G., Maciejewski, P. K., \& Rosenheck, R. A. (1999). The effects of marital dissolution and marital quality on health and health service use among women. Medical Care, 37(9), 858-873.

Rodríguez, D. C. (1997). Entre el mito y la experiencia vivida: las jefas de familia. En: González, S. y Tuñón, S. (comp.). Familias y mujeres en México. México: El Colegio de México, $1^{\text {a }}$ ed., pp. 195-238.

Rosenberg, M. (1989). Society and the adolescent self-image (Revised edition). Middletown, C. T.: Wesleyan University Press.

Ross C. \& Mirowsky, J. (1989). Explaining the social patterns of depression: control and problem solving - or support and talking? Journal of Health and Social Behavior, 30(2), 206-219.

Russel, D. W. \& Cutrona, C. E. (1991). Social support, stress, and depressive symptoms among the elderly: test of a process model. Psychology of Aging, 6, 190-201.
Sherbourne, C. D. \& Stewart, A. L. (1991). The MOS social support survey. Social Science \& Medicine, 32, 705-712.

Valdés, Y. (2004). Impacto psicológico del divorcio en la mujer. Una nueva visión de un viejo problema. Biblioteca virtual CLASCO.

Vega, W. A., Kolody, B., Valle, R. \& Weir, J. (1991). Social networks, social support and their relationship to depression among immigrant Mexican women. Human Organization, $50(2), 154-162$.

Verbrugge, L. (1979). Marital status and health. Journal of Marriage and the Family, 41(2), 267-285.

Waldron, I., Hughes, M. E., \& Brooks, T. L. (1996). Marriage protection and marriage selection prospective evidence for reciprocal effects of marital status and health. Social Science \& Medicine, 43, 113-123.

Wallerstein, J. S. \& Kelly, J. B. (1975). The effects of parental divorce: Experiences of the preschool child. Journal of the American Academy of Child Psychiatry, 14, 600-616.

Weinraub, M. \& Wolf, B. M. (1983). Effects of Stress and Social Supports on Mother-Child Interactions in Single and TwoParent Families. Child Development, 54, 1297-1311.

Wille, D. E. (1998). Longitudinal analysis of 'mothers' and fathers' responses on the Maternal Separation Anxiety Scale. Merrill-Palmer Quarterly, 44, 216-233.

Zung, W. W. K., Broadhead, E., \& Roth, M. E. (1993). Prevalence of depressive symptoms in primary care, Journal of family practice, 37(4), 337-344.

Zúñiga, M. \& Ribeiro, M. (2005). Divorcio y solidaridad familiar. En Landero, R. (ed.). (2005). Ruptura conyugal y monoparentalidad. Dificultades afrontadas y apoyo social, 63-90. Facultad de Psicología, UANL. 